\title{
Genotyping-by-sequencing enables linkage mapping in three octoploid cultivated strawberry families
}

Kelly J Vining Corresp., 1 , Natalia Salinas ${ }^{2}$, Jacob A Tennessen ${ }^{3}$ ， Jason D Zurn ${ }^{4}$ ， Daniel James Sargent ${ }^{5,6}$, James Hancock $^{7}$, Nahla V Bassil ${ }^{1,4}$

1 Department of Horticulture, Oregon State University, Corvallis, Oregon, United States

2 Department of Horticulture, University of Florida, Wimauma, Florida, United States

3 Department of Integrative Biology, Oregon State University, Corvallis, Oregon, United States

4 National Clonal Germplasm Repository, United States Department of Agriculture, Agricultural Research Service, Corvallis, Oregon, United States

5 Research and Innovation Centre, Fondazione Edmund Mach,, San Michele all'Adige, Italy

6 East Malling Enterprise Centre, Driscoll's Genetics Limited, East Malling, United Kingdom

7 Department of Horticulture, Michigan State University, East Lansing, Michigan, United States

Corresponding Author: Kelly J Vining

Email address: kelly.vining@oregonstate.edu

Genotyping-by-sequencing (GBS) was used to survey genome-wide single-nucleotide polymorphisms (SNPs) in three biparental strawberry (Fragaria xananassa) populations with the goal of evaluating this technique in a species with a complex octoploid genome. GBS sequence data were aligned to the $F$. vesca ' $F v b$ ' reference genome in order to call SNPs. Numbers of polymorphic SNPs per population ranged from 1,163 to 3,190. Linkage maps consisting of 30-65 linkage groups were produced from the SNP sets derived from each parent. The linkage groups covered $99 \%$ of the Fvb reference genome, with three to seven linkage groups from a given parent aligned to any particular chromosome. A phylogenetic analysis performed using the POLiMAPS pipeline revealed linkage groups that were most similar to ancestral species $F$. vesca for each chromosome. Linkage groups that were most similar to a second ancestral species, F. iinumae, were only resolved for Fvb 4. The quantity of missing data and heterogeneity in genome coverage inherent in GBS complicated the analysis, but POLiMAPS resolved $F$. xananassa chromosomal regions derived from diploid ancestor $F$. vesca. 
1 Genotyping-by-sequencing enables linkage mapping in three octoploid cultivated

2 strawberry families

3 Kelly J. Vining ${ }^{1}$, Natalia Salinas ${ }^{2}$, Jacob A. Tennessen ${ }^{3}$, Jason D. Zurn ${ }^{4}$, Daniel James Sargent ${ }^{6 *}$,

4 James Hancock ${ }^{7}$, and Nahla V. Bassil ${ }^{1,4}$

$5 \quad{ }^{1}$ Department of Horticulture, Oregon State University, Corvallis, Oregon 97331 USA

62 Department of Horticulture, University of Florida, Wimauma, Florida 33598 USA

$7 \quad{ }^{3}$ Department of Integrative Biology, Oregon State University, Corvallis, Oregon 97331 USA

$8{ }^{4}$ United States Department of Agriculture, Agricultural Research Service, National Clonal

9 Germplasm Repository, Corvallis, Oregon, 97333 USA

$10{ }^{6}$ Fondazione Edmund Mach, Research and Innovation Centre, via Mach 1, San Michele

11 all'Adige 38010 (TN), Italy

$12{ }^{7}$ Department of Horticulture, Michigan State University, East Lansing, Michigan, 48824 USA

13 *Present Address: Driscoll's Genetics Limited, East Malling Enterprise Centre, New Road, East 14 Malling, Kent ME19 6BJ, UK

\section{ABSTRACT}

Genotyping-by-sequencing (GBS) was used to survey genome-wide single-nucleotide polymorphisms (SNPs) in three biparental strawberry (Fragaria $\times$ ananassa) populations with the goal of evaluating this technique in a species with a complex octoploid genome. GBS sequence data were aligned to the $F$. vesca ' $F v b$ ' reference genome in order to call SNPs. Numbers of polymorphic SNPs per population ranged from 1,163 to 3,190. Linkage maps consisting of 30-65 linkage groups were produced from the SNP sets derived from each parent. The linkage groups covered $99 \%$ of the $F v b$ reference genome, with three to seven linkage groups from a given parent aligned to any particular chromosome. A phylogenetic analysis performed using the POLiMAPS pipeline revealed linkage groups that were most similar to ancestral species $F$. vesca for each chromosome. Linkage groups that were most similar to a second ancestral species, $F$. iinumae, were only resolved for $F v b 4$. The quantity of missing data and heterogeneity in genome coverage inherent in GBS complicated the analysis, but POLiMAPS resolved $F$. ×ananassa chromosomal regions derived from diploid ancestor $F$. vesca.

\section{BACKGROUND}


Genotyping-by-sequencing (GBS) is a powerful, cost-effective method for identifying single-nucleotide polymorphisms (SNPs) on a whole-genome scale. The GBS technique commonly used involves a form of reduced representation genome sequencing based on partial restriction enzyme digestion, usually with a methylation-sensitive restriction enzyme, followed by barcoded adaptor ligation and next-generation sequencing of highly multiplexed samples, typically 48, 96, or 384 samples per lane (Elshire et al., 2011; Davey et al., 2011). Applications of GBS range from germplasm diversity and population structure assessment to molecular marker discovery. The high throughput and low per-sample cost of GBS makes it an attractive option for plant breeding populations, as it can be used to saturate genetic maps (Russell et al., 2014, Ward et al., 2013), perform QTL mapping and genome-wide association analyses (GWAS) for traits of interest (Spindel et al., 2013), and enable genomic selection (Spindel et al., 2015). GBS has been applied to polyploid crop plants, including oat (Huang \& Han, 2014), blueberry (McCallum et al., 2016), and wheat (Poland et al., 2012; Saintenac et al., 2013).

The genus Fragaria consists of 20 species that range in ploidy from diploid to decaploid (Liston et al. 2014). The polyploid species have complex evolutionary histories, including hybridization events and chromosomal rearrangements (Njuguna et al., 2013; Liston, Cronn \& Ashman, 2014; Tennessen et al., 2014). The cultivated strawberry, Fragaria $\times$ ananassa Duch. ex Rozier, has 28 pairs of chromosomes and is a recent allo-octoploid $(2 \mathrm{n}=8 \mathrm{x}=56)$, having arisen in Europe in the $18^{\text {th }}$ century from hybridization between two octoploids: North American F. virginiana Mill. and South American F. chiloensis (L.) Duchesne ex Weston (Liston, Cronn \& Ashman, 2014). Phylogenetic analysis of octoploid Fragaria species have been conducted using nuclear genes (Rousseau-Gueutin et al., 2009; DiMeglio et al., 2014), almost complete chloroplast genomes (Njuguna et al., 2013; Govindarajulu et al., 2015), and genome-wide markers (Tennessen et al., 2014; Govindarajulu et al., 2015; Qiao et al., 2016). These studies support a model in which the octoploid Fragaria genome contains four ancestral sub-genomes. One of the four sub-genomes appears to have originated from $F$. vesca $\mathrm{L}$., one from $F$. iinumae Makino, and two from an unknown ancestor phylogenetically close to $F$. iinumae (Tennessen et al., 2014; Sargent et al., 2016). Two high-throughput genotyping platforms have been recently developed for $F . \times$ ananassa: a 90K Affymetrix Axiom array containing 95,062 marker loci (Bassil et al., 2015), and two microarrays based on Diversity Array Technology (DArT) markers (Sánchez-Sevilla et al., 2015). 
63

64

65

66

67

68

69

Linkage mapping has proved to be a useful tool for Fragaria genomics. The genome of the diploid $F$. vesca was assembled using an SSR-based map (Sargent et al., 2012) and subsequently improved with dense targeted capture maps comprising over 9,000 polymorphisms (Tennessen et al., 2014). These diploid linkage maps have allowed traits such as sex determination to be mapped (Ashman et al., 2015; Tennessen et al., 2016). While the similarity of Fragaria sub-genomes has presented challenges for genetic linkage mapping in breeding populations of octoploid $F$. ×ananassa, linkage maps have been applied to resolve genomic structure and identify chromosomal rearrangements (Sargent et al., 2012, 2016; Isobe et al., 2013; Tennessen et al., 2014; Davik et al., 2015; Sánchez-Sevilla et al., 2015), as well as to map traits (Spigler \& Ashman, 2011; Zorrilla-Fontanesi et al., 2011; Molina-Hidalgo et al., 2013; Tennessen et al., 2016). Despite their recent polyploid origin, inheritance in the octoploid Fragaria species is primarily disomic (Bringhurst, 1990; Lerceteau-Köhler et al., 2003; Rousseau-Gueutin et al., 2008), allowing distinct linkage groups to be constructed and assessed.

The objectives of this study were to 1) evaluate the utility of GBS by developing linkage maps for three bi-parental $F$. ×ananassa populations using SNP markers derived from GBS; and 2) test the efficacy of the POLiMAPS pipeline in resolving sub-genome contributions from the ancestral diploid Fragaria species.

MATERIALS \& METHODS

\section{Plant Material, DNA Extraction and Quantitation}

The strawberry samples analyzed in this study consisted of: parents and 24 offspring from the 'Holiday' $\times$ 'Korona' population from the Netherlands (van Dijk et al., 2014); parents and 60 seedlings from the 'Tribute' $\times$ 'Honeoye' population from Michigan State University (MSU) (Castro et al., 2015; Sooriyapathirana et al., 2015); parents and 51 offspring from the 'Redgauntlet' × 'Hapil' population from East Malling Research, UK (Sargent et al., 2012; Supplemental Table 1). DNA was extracted from actively growing leaf tissue with the E-Z 96® Plant DNA extraction kit (Omega BioTek, Norcross, GA, USA) as previously described (Gilmore, Bassil \& Hummer, 2011). The resulting genomic DNA was quantitated with the Quant-iT ${ }^{\mathrm{TM}}$ Picogreen ${ }^{\circledR}$ Assay (Invitrogen, Eugene, OR, USA) according to the manufacturer's 
92 recommendations using a Victor ${ }^{3} \mathrm{~V} 1420$ Multilabel Counter (Perkin Elmer, Downers Grove, IL, 93 USA). The DNA concentration was adjusted to $100 \mathrm{ng} / \mu \mathrm{L}$ per sample for subsequent genotyping 94 by sequencing (GBS) library preparation.

95

96

97

98

GBS Library Preparation

Preliminary testing with three restriction enzymes (PstI, MspI and ApeK1) for fragment size range led to selection of ApeKI for GBS library construction. Three GBS libraries were constructed at the USDA-ARS National Clonal Germplasm Repository (NCGR) and one was constructed at Clemson University according to the procedure previously described (Elshire et al., 2011) for 96 samples using DNA (100 ng per sample) digested with 4 U of ApeKI (New England Biolabs, Ipswich, MA, USA). The annealed and normalized unique and four-nucleotidebarcoded adaptors were obtained from Clemson University Genomics Institute (CUGI) and from the Oregon State University (OSU) Center for Genome Research and Biocomputing (CGRB) core facility. Two libraries were sequenced at the CGRB, one at CUGI, and one at the North Carolina State University Genomic Sciences Laboratory (Supplemental Table 1). At each of these labs, libraries were quantitated with a Qubit ${ }^{\circledR}$ fluorometer (Invitrogen, Carlsbad, CA, USA), checked for adequate size distribution ( 150 - $350 \mathrm{bp}$ ) with the Bioanalyzer 2100 HSDNA chip (Agilent Technologies, Santa Clara, CA, USA), and sequenced with the Illumina HiSeq2000 (101 bp, single-end).

\section{Genotyping}

Adapter sequences were removed at the sequencing facilities prior to our receiving the data. Reads were quality-filtered by converting to ' $N$ ' sites with Phred-scaled quality scores lower than 20, and by excluding reads with fewer than 50 retained (high-quality) sites. SNPs were called using the POLiMAPS pipeline (Tennessen et al., 2014). In brief, sequence reads were aligned to the $F v b$ genome assembly (Tennessen et al., 2014) using BWA version 0.7.12 with parameter -n 0.001 (Li and Durbin 2009). SAMtools version1.1 was used (Li et al., 2009) to generate a pileup format file for each of the three crosses and a custom Perl script was used to call polymorphisms (available at https://github.com/listonlab/POLiMAPS). POLiMAPS 
121 identifies markers with approximately Mendelian segregation by requiring a minimum number

122 of offspring displaying each of the two possible genotypes (parameter $-\mathrm{o}$, default $=8$ ). It also 123 sets a maximum value for number of offspring with missing genotypes (parameter $-\mathrm{m}$, default $=$

124 1). Default parameters were used with the following exceptions. Because there were relatively 125 few offspring in the 'Holiday' $\times$ 'Korona' cross (24), we decreased -0 to 6 . Conversely, because 126 there were more offspring in 'Redgauntlet' $\times$ 'Hapil' (51) and 'Tribute' $\times$ 'Honeoye' (63), we 127 increased $-\mathrm{m}$ to 4 for 'Redgauntlet' $\times$ 'Hapil' and to 5 for 'Tribute' $\times$ 'Honeoye'.

Linkage Mapping

SNPs that were segregating in both parents were excluded from linkage mapping, as tri-

Phylogenetic Analysis or quad-allelic markers were expected to be rare, and difficult to distinguish from sequencing errors. Segregating loci were organized into parental sets, which were subjected to linear regression mapping using JoinMap ${ }^{\circledR}$ V. 4.1 (Van Ooijen, 2006). A minimum independence logarithm of odds (LOD) threshold of 3 was used for establishing the linkage groups (LG).

Dendrograms were constructed for each linkage group using the genetic information for each cultivar and the diploid congeners following the previously described POLiMAPS approach for octoploid Fragaria (Tennessen et. al. 2014). This method identified Illumina reads containing markers associated with a linkage group, which were then assigned to that chromosome, and treated all other sites on those reads as potential phylogenetic characters. For each of the seven haploid Fragaria chromosomes, a phylogeny was generated with each parent expected to provide distinct linkage groups representing the four sub-genomes. The $F v b$ reference genome of $F$. vesca was used along with whole genome data from the same diploid samples as in Tennessen et al. (2014): F. mandshurica, F. bucharica, F. viridis, F. nipponica, $F$. iinumae, and Rubus coreanus as an outgroup. RAxML was used with -N autoMRE and -m GTRCAT and 100 bootstrap replicates to estimate separate phylogenies for each of the seven haploid Fragaria chromosomes (Stamatakis, 2006). Two rounds of analysis using the same protocol were performed. In the first round, chromosome information from four diploids $(F$. 
150 vesca, $F$. mandshurica, $F$. viridis, and $F$. iinumae) and the $F$. ×ananassa linkage groups which 151 aligned to the homologous chromosome and had at least 300 phylogenetic sites were included. 152 The objective of this analysis was to identify the LG from each population that belonged to each 153 of the sub-genomes. When more than four linkage groups per parental map were found, an 154 attempt was made to merge some of the linkage groups in each parental map using the following 155 criteria: 1) continuous marker position on the $F v b$ reference; 2) a Chi squared $\left(\chi^{2}\right)$ test supporting 156 linkage between the last SNP at the end of one linkage group and the first SNP at the beginning 157 of another linkage group $(\mathrm{P}<0.05) ; 3)$ similar phylogenetic position of the two linkage groups; and 4) strongest cross-link (SCL) metric between JoinMap groups.

Once some of the LGs were merged, the phylogenetic analysis was repeated, with the additional Fragaria comparators F. nipponica and F. bucharica, and with Rubus coreanus to serve as an outgroup. The minimum number of phylogenetic sites per JoinMap linkage group was lowered to 150 for all parents and sites were allowed to be missing in any of the diploids.

\section{'Holiday' Chromosome 6 Comparison between GBS and Axiom Array Data}

An integrated linkage map for 'Holiday' chromosome 6D was created utilizing the 90K Axiom data (Bassil et al., 2015) and the GBS data from 'Holiday' groups 2 and 3 via a graphical

167

168

169

170

171

172

173

174

175

176

177

178

179 mapping approach to further assess the quality of the GBS data. Bowtie2 version 2.2.9 (Langmead et al., 2009) and SAMtools version 1.3.1 (Li et al., 2009) were used to align the Axiom 'Holiday' chromosome 6D map and the integrated 'Holiday' 6D map to the $F v b$ assembly to visualize genetic rearrangements between $F$. vesca ssp. bracteata and $F$. ×ananassa.

\section{RESULTS}

\section{Genotyping}

The yield of high-quality GBS data obtained from the parents, defined as Illumina reads containing both the expected barcode sequence and ApeKI cut site remnant, ranged from $98 \mathrm{Mb}$ to $185 \mathrm{Mb}$ (Table 1). The number of distinct loci with at least one high coverage $(\geq 32 \times)$ site per parent ranged from 7,058 to 12,395, and the number distinct loci with at least 64 high coverage sites (the length of a trimmed read) per parent ranged from 4,119 to 9,117 (Supplementary Table 1). Numbers of reads that aligned to the $F v b$ reference genome ranged from 992,053 ('Hapil') to 
180

181

182

183

184

185

186

187

188

189

190

191

192

193

194

195

196

197

198

199

200

201

202

203

204

205

206

207

208

1,754,838 ('Honeoye'; Table 1). Numbers of SNPs, which were identified by POLiMAPS from reads aligning to the $F v b$ reference genome, are also listed in Table 1. The greatest number of SNPs was found in the 'Redgauntlet' $\times$ 'Hapil' population $(3,190)$. In the 'Holiday' $\times$ 'Korona' population, the number of SNPs was 2,136. The fewest SNPs were found in the 'Tribute' $\times$ 'Honeoye' population $(1,163)$.

Progeny plants were categorized as having a high quantity of missing data when the number of SNP sites lacking a defined genotype was greater than or equal to $10 \%$. Missing data for linkage mapping was estimated only with respect to segregating genotypes on each individual sub-genome; thus, for example, if only one sub-genome had a segregating SNP at a particular locus and we did not identify reads from all four sub-genomes at that locus, we would not consider that to be missing data. The smallest population, 'Holiday' $\times$ 'Korona', had only one plant with 10\% missing SNPs. In the 'Redgauntlet' $\times$ 'Hapil' population, two offsprings had greater than or equal to $10 \%$ missing data: $11 \%$ and $27 \%$, the latter of which was excluded from JoinMap analysis. In the largest population, 'Tribute' $\times$ 'Honeoye', seven plants had greater than or equal to $10 \%$ missing data, and four of these were excluded from further analysis because they had greater than or equal to $30 \%$ missing data.

\section{Linkage Mapping and Homolog Assignment}

The number of JoinMap linkage groups obtained from parental genotypes ranged from 30 ('Tribute') to 65 ('Holiday'). Over all three populations, there were 178 JoinMap groups, with 46 (26\%) consisting of fewer than ten SNPs, and 25 with gaps of $15 \mathrm{cM}$ or larger. For a given chromosome, the number of aligned linkage map groups ranged from three to seven (Fig. 1, Table 2). For the 'Tribute' $\times$ 'Honeoye' cross, a total of 29 chromosome-aligned groups were constructed from 'Tribute'-derived SNPs, and 33 were constructed from 'Honeoye'-derived SNPs. For the 'Holiday' $\times$ 'Korona' cross, 38 groups were constructed from 'Holiday'-derived SNPs, and 49 from 'Korona'-derived SNPs. For the 'Redgauntlet' × 'Hapil' cross, 39 groups were constructed from 'Redgauntlet'-derived SNPs, and 37 were from 'Hapil'-derived SNPs. The number of SNPs on chromosome-aligned linkage groups ranged from 21 to 169 (Fig. 2). Altogether, the linkage groups covered $99 \%$ of the $F v b$ genome.

\section{Sub-genome Assignment}


A phylogenetic analysis was performed in order to identify distinct linkage groups

210

211

212

213

214

215

216

217

218

219

220

221

222

223

224

225

226

227

228

229

230

231

232

233

234

235

236

237

238 representing ancestral sub-genomes. Since linkage groups pertaining to the same reference chromosome were aligned together, for phylogenetic purposes missing data was calculated across sub-genomes. Thus, for the diploid Fragaria species, there was a median of 8\% missing data in the phylogenetic character matrix (mean $=16 \%$, range $=4-53 \%)$, and for linkage groups there was a median of $95 \%$ missing data in the phylogenetic character matrix (mean $=94 \%$, range $=81-99 \%$ ). The resulting trees - one for each chromosome - were examined to identify JoinMap groups that could potentially be merged according to proximity on a tree. Merging reduced the overall number of chromosome-aligned groups by six or fewer for 'Tribute' (from 29 to 27), 'Honeoye' (from 33 to 28), 'Redgauntlet' (from 39 to 34), and 'Hapil' (from 37 to 31; Table 2). The number of 'Holiday' groups was reduced by nine (from 38 to 29), and the number of 'Korona' groups was reduced by 17 (from 49 to 32 ).

Once JoinMap groups were merged, a second round of phylogenetic analysis was performed to produce a final set of trees, with one representing each $F v b$ chromosome (Fig. 3, Supplemental Files 1-3). Most linkage groups aligned uniquely to $F v b$ chromosomes. However, 'Holiday'-derived linkage group 8, which consisted of 32 SNPs and had a length of $77.1 \mathrm{cM}$, aligned to two $F v b$ chromosomes: $F v b 3$ and $F v b 6$ (Supplemental File 1). Group 8 encompassed the entire Fvb3 chromosome, and also the last approximately $3.7 \mathrm{Mb}$ of $F v b 6$.

Clear $F$. vesca-like clades were distinguishable on just two of the chromosomal cladograms: $F v b 1$ and $F v b 2$. All six parental genotypes were represented in the $F$. vesca clade on $F v b$ 1, and only 'Holiday' was absent from the $F$. vesca clade on $F v b 2$ (Table 3). On the $F v b$ 7 cladogram, $F$. vesca and the other Fragaria species were not clearly differentiated. An $F$. iinumae-like clade was only distinguishable in the $F v b 4$ cladogram.

\section{'Holiday' Chromosome 6 Comparison between GBS and Axiom Array Data}

An integrated map for 'Holiday' chromosome 6D was created using marker data from the $90 \mathrm{~K}$ Axiom array for the 'Holiday' $\times$ 'Korona' population (Bassil et al., 2015). Three individuals of the 23 used in the initial mapping in the present study were excluded either due to a large amount of missing Axiom data (H-02552) or inconsistencies between the Axiom data and GBS data for the samples (H-02572 and H-02637). When the GBS and Axiom markers were mapped together, an $83 \mathrm{cM}$ linkage group was produced, where markers co-segregated into 15 
239 bins (Fig. 4, Supplementary Table 2). The graphical mapping approach was able to show the

240 integration of the GBS data and recombination events were easy to visualize (Supplementary

241 Table 2). Moreover, the total length of the integrated map is very similar to the $95.6 \mathrm{cM}$ map

242 produced by (Bassil et al., 2015) and the shared marker order did not vary, demonstrating the

243 quality of the GBS data. Map resolution was considerably lower in the integrated map due to the 244 reduced population size.

245 Many of the same major chromosomal rearrangements between $F$. vesca ssp. bracteata

246 and $F$. ×ananassa were observed between the integrated GBS map and the $90 \mathrm{~K}$ Axiom-derived

247 map (Fig. 4). The Axiom-derived map was able to identify a few more micro rearrangements

248 than the integrated map. This is to be expected as the population size used to construct the

249 Axiom-derived map was much larger than the integrated map. Many of the rearrangements

250 observed were a few markers rather than large blocks (Fig. 4). As such it is unknown if the

251 rearrangements observed are mapping or assembly errors or if the rearrangements are due to

252 evolutionary differences between $F$. vesca ssp. bracteata and $F$. ×ananassa

253

254

255

256

257

258

259

260

261

262

263

264

265

266

267

\section{DISCUSSION}

GBS and related reduced-representation sequencing methods have recently been used to study F. iinumae (Mahoney et al., 2016), and F. ×ananassa (Davik et al., 2015). Both of those studies employed protocols using two different restriction enzymes. The use of two restriction enzymes in which one enzyme is a less-frequent cutter further reduces the sequenced fraction of the genome because only genome fragments containing the less-frequent site and a morefrequent site are selected. This has the effect of increasing overall sequencing depth and likelihood of SNP detection over covered regions. In the present study, high-quality SNP data was obtained for three octoploid, biparental $F$. ×ananassa using a single restriction enzyme, ApeKI as previously done in many plant species including blueberry (McCallum et al., 2016) and red raspberry (Ward et al., 2013). The POLiMAPS pipeline (Tennessen et al., 2014b) was used to identify sub-genomes derived from $F$. vesca (Av), and $F$. iinumae (Bi). The remaining two sub-genomes, B1 and B2, could not be distinguished and were un-assigned and referred to as B12. The Av sub-genome corresponds to sub-genome A of van Dijk et al., (2014) who first 
268 denoted the sub-genomes based on their divergence from $F$. vesca as A, B, C and D, in order of 269 most to least divergence.

270 The numbers of polymorphic SNPs obtained from 'Redgauntlet' $\times$ 'Hapil' and 'Holiday'

$271 \times$ 'Korona' were in the range of those obtained for $F$. iinumae and $F$. ×ananassa in the

272 aforementioned experiments. However, the numbers of polymorphic SNPs used for mapping

273 varied widely among the three analyzed populations. The 'Redgauntlet' $\times$ 'Hapil' population had

274 by far the greatest number of polymorphic SNPs, with 1,253 and 1,096 derived from each

275 parental genotype, while the 'Holiday' $\times$ 'Korona' population had 735 and 1,096, and the

276 'Tribute' $\times$ 'Honeoye' population had 358 and 468 SNPs. Relative levels of homozygosity may

277 account for this variability. The number of restriction enzymes employed, choice of restriction

278 enzymes, and sequence coverage depth per individual over the sampled portion of the genome

279 can also affect the number of polymorphic SNPs detected (Glaubitz et al., 2014). Interestingly,

280 the 'Tribute' $\times$ 'Honeoye' population had the highest number of reads aligned to the $F$. vesca

281 reference genome, yet it had the lowest number of SNP calls and the highest quantity of missing

282 data. The lower number of SNP calls may indicate low genetic variation within the parents.

Many of the JoinMap groups that aligned to the $F v b$ chromosomes were small, consisting of fewer than ten SNPs. Others were longer, but had large gaps $(>15 \mathrm{cM})$. Homozygous genome regions can account for large gaps in linkage maps, and the fragmented linkage groups can be attributed to heterogeneity in genome coverage of GBS data, which results in an overall high quantity of missing data. Low marker density and large gaps can also result from conserved chromosomal regions with high homozygosity. 'Holiday' and 'Korona' have common ancestors in their pedigrees, making regions of shared homozygosity more likely in that population (van Dijk et al., 2014). A study of simple sequence repeat (SSR) markers in the 'Redgauntlet' $\times$ 'Hapil' population reported six regions on five linkage groups with $>20 \mathrm{cM}$ gaps (Sargent et al., 2012), however, marker density in that study was overall much lower than in any of the datasets analyzed here and the power to bridge these gaps was much larger due to the increased family sizes used.

The numbers of SNPs mapped by GBS in this study ranged from 2,663 in the 'Tribute' $\times$ 'Honeoye' population to 3,912 in 'Redgauntlet' $\times$ 'Hapil'. When using the Axiom ${ }^{\circledR}$ IStraw90 Axiom Array, the number of SNPs mapped were $>6,500$ in 'Holiday' $\times$ 'Korona' (Bassil et al., 
298

299

300

301

302

303

304

305

306

307

308

309

310

311

312

313

314

315

316

317

318

319

320

321

322

323

324

325

326

327

328

2015), > 8,400 in 'Darselect' × 'Monterey' (Sargent et al., 2016), and 11,002 in Reikou (Nagano et al., 2017). At this time, the availability of a high throughput array for the octoploid domestic strawberry appears to meet the need for linkage mapping and has generated a larger number of mapped markers when compared to the GBS approach used in this study. This array was shown to be useful for mapping in F. iinumae (Mahoney et al., 2016), and in F. vesca (Shields \& Davis, 2017), but has not yet been applied to other Fragaria species. Cost per sample for GBS is considered an advantage over array-based genotyping. However, the availability of the Axiom ${ }^{\circledR}$ IStraw35 384HT array at a cost of $\$ 50$ per sample has decreased the price of high throughput genotyping which now is closer to that of GBS. The IStraw35 array is expected to be just as useful as the IStraw90 for linkage mapping in F. ×ananassa but its usefulness in other species awaits further evaluation. However, since SNPs in both arrays targeted SNPs in the octoploid domestic strawberry and were enriched for the $F$. vesca sub-genome (Sargent et al., 2016) the arrays are not expected to be as useful in other wild species while GBS can be applied to any species.

The goal of the POLiMAPS phylogenetic analysis was two-fold: First, to resolve homoeologs in cases where more than four JoinMap linkage groups from any crossing parent aligned to the same haploid reference chromosome; and second, to identify ancestral diploid subgenome contributions. Comparative genomic mapping between octoploid and diploid Fragaria species has reported synteny and high colinearity along chromosomes (Davik et al., 2015; van Dijk et al., 2014). The inclusion of more than one diploid Fragaria species in the phylogenetic analysis increased the robustness of the $F$. vesca clade. We expected all sub-genomes to have numerous regions sufficiently similar to the reference genome to produce correctly-aligning reads, given the previous success of this alignment pipeline (Tennessen et al., 2014). However it is likely that a higher proportion of reads from the $F$. vesca sub-genome aligned given its higher similarity to the reference genome, leading to an enrichment for $F$. vesca sub-genome markers in our analysis. Because one of our goals was to connect linkage groups with $F v b$ chromosomes, we prioritized aligning reads with high confidence to the reference genome, and discarded reads that could not align given the BWA -n 0.001 parameter. Other analyses of GBS data in Fragaria may seek to maximize the number of markers even if their reference genome position is less certain. When high-quality assemblies of the $F . \times$ ananassa sub-genomes, and of other Fragaria diploids, become available, the issue of aligning divergent reads will be less of a concern. 
329 Overall, the greatest number of SNPs were assigned to the $F$. vesca sub-genome. Each parental

330 genotype had linkage groups that could be assigned to the $F$. vesca sub-genome on chromosome

$331 F v b$ 1, and all parental genotypes except for 'Holiday' had $F$. vesca-like groups on chromosomes

$332 F v b 2$ and $F v b$ 7. Fvb 4 was the only chromosome for which all parental genotypes had linkage

333 groups that could be assigned to the $F$. iinumae sub-genome, however, most of the linkage

334 groups from parental genotypes could not be assigned to any sub-genome. These results are

335 consistent with the findings of Hirakawa et al. (2013), who aligned 57\% of the scaffolds in an $F$.

$336 \times$ ananassa assembly to $F$. vesca pseudomolecules, and concluded that approximately $20 \%$ of the

337 scaffolds were $F$. ×ananassa-specific.

One linkage group derived from 'Holiday' aligned to both chromosome 3 and

chromosome 6. This region may represent a translocation event. Markers from the distal end of

$F v b$ 6, comprising over $3 \mathrm{Mb}$ and over 700 genes, are included in a linkage group containing

markers from across $F v b 3$. This gene-dense region of $F v b 6$ contains genes and QTLs linked to important traits, such as sex phenotype (Goldberg, Spigler \& Ashman, 2010; Ashman et al., 2015). However, given the small sample size of the 'Holiday' $\times$ 'Korona' cross, this could also be a spurious association.

In summary, POLiMAPS was employed with genotyping-by-sequencing data in three small families to resolve $F$. ananassa chromosomal regions derived from the diploid $F$. vesca. However, the large number of missing data in GBS experiments, combined with the complex relationships among homoeologs in polyploid plants, complicate such analyses and may limit the usefulness of GBS in these plants. Furthermore, the availability of array-based high throughput genotyping at a reduced cost in the form of the IStraw35 array provides another useful and easy tool for linkage mapping in $F$. ×ananassa. Use of an $F$. ×ananassa reference sequence for SNP detection and higher coverage of GBS libraries developed after cutting with two restriction endonucleases may address the challenges observed in this study and require further evaluation.

DATA AVAILABILITY

The raw sequence data from this project is available via the NCBI Sequence Read Archive, ftp://ftp.bioinfo.wsu.edu/species/Fragaria_vesca/Fvesca-genome.v2.0.a1/. 
360

361

362

363

364

365

366

367

368

369

370

371

372

373

374

375

376

377

378

379

380

381

382

383

384

385

386

387

388

389

390

391

392

393

\section{ACKNOWLEDMENTS}

We thank Eric van de Weg at Wageningen Plant Research, The Netherlands, for helpful suggestions and input.

\section{References}

Ashman T-L., Tennessen JA., Dalton RM., Govindarajulu R., Koski MH., Liston A. 2015. Multilocus Sex Determination Revealed in Two Populations of Gynodioecious Wild Strawberry, Fragaria vesca subsp. bracteata. G3: Genes|Genomes|Genetics 5:27592773. DOI: $10.1534 / \mathrm{g} 3.115 .023358$.

Bassil NV., Davis TM., Zhang H., Ficklin S., Mittmann M., Webster T., Mahoney L., Wood D., Alperin ES., Rosyara UR., Koehorst-vanc Putten H., Monfort A., Sargent DJ., Amaya I., Denoyes B., Bianco L., van Dijk T., Pirani A., Iezzoni A., Main D., Peace C., Yang Y., Whitaker V., Verma S., Bellon L., Brew F., Herrera R., van de Weg E. 2015. Development and preliminary evaluation of a 90 K Axiom ${ }^{\circledR}$ SNP array for the allooctoploid cultivated strawberry Fragaria ×ananassa. BMC Genomics 16:155. DOI: 10.1186/s12864-015-1310-1.

Bringhurst RS. 1990. Cytogenetics and evolution in American Fragaria. HortScience 25:879881.

Davey JW., Hohenlohe PA., Etter PD., Boone JQ., Catchen JM., Blaxter ML. 2011. Genomewide genetic marker discovery and genotyping using next-generation sequencing. Nature Reviews Genetics 12:499-510. DOI: 10.1038/nrg3012.

Davik J., Sargent DJ., Brurberg MB., Lien S., Kent M., Alsheikh M. 2015. A ddRAD Based Linkage Map of the Cultivated Strawberry, Fragaria xananassa. PLOS ONE 10:e0137746. DOI: 10.1371/journal.pone.0137746.

van Dijk T., Pagliarani G., Pikunova A., Noordijk Y., Yilmaz-Temel H., Meulenbroek B., Visser RG., van de Weg E. 2014. Genomic rearrangements and signatures of breeding in the allo-octoploid strawberry as revealed through an allele dose based SSR linkage map. BMC Plant Biology 14:55. DOI: 10.1186/1471-2229-14-55.

DiMeglio LM., Staudt G., Yu H., Davis TM. 2014. A phylogenetic analysis of the genus Fragaria (strawberry) using intron-containing sequence from the ADH -1 gene. PLOS ONE 9:e102237. DOI: 10.1371/journal.pone.0102237.

Elshire RJ., Glaubitz JC., Sun Q., Poland JA., Kawamoto K., Buckler ES., Mitchell SE. 2011. A robust, simple Genotyping-by-Sequencing (GBS) approach for high diversity species. PLoS ONE 6:e19379. DOI: 10.1371/journal.pone.0019379. 
402

403

404

405

406

407

408

409

410

411

412

413

414

415

416

417

418

419

420

421

422

423

424

425

426

427

428

429

430

431

432

Gilmore BS., Bassil NV., Hummer KE. 2011. DNA extraction protocols from dormant buds of twelve woody plant genera. Journal of the American Pomological Society 65:201-206.

Glaubitz JC., Casstevens TM., Lu F., Harriman J., Elshire RJ., Sun Q., Buckler ES. 2014. TASSEL-GBS: A high capacity Genotyping by Sequencing analysis pipeline. PLoS ONE 9:e90346. DOI: 10.1371/journal.pone.0090346.

Goldberg MT., Spigler RB., Ashman T-L. 2010. Comparative genetic mapping points to different sex chromosomes in sibling species of wild strawberry (Fragaria). Genetics 186:1425-1433. DOI: 10.1534/genetics.110.122911.

Govindarajulu R., Parks M., Tennessen JA., Liston A., Ashman T-L. 2015. Comparison of nuclear, plastid, and mitochondrial phylogenies and the origin of wild octoploid strawberry species. American Journal of Botany 102:544-554. DOI: 10.3732/ajb.1500026.

Hirakawa H., Shirasawa K., Kosugi S., Tashiro K., Nakayama S., Yamada M., Kohara M., Watanabe A., Kishida Y., Fujishiro T., Tsuruoka H., Minami C., Sasamoto S., Kato M., Nanri K., Komaki A., Yanagi T., Guoxin Q., Maeda F., Ishikawa M., Kuhara S., Sato S., Tabata S., Isobe SN. 2013. Dissection of the octoploid strawberry genome by deep sequencing of the genomes of Fragaria species. DNA Research:dst049. DOI: 10.1093/dnares/dst049.

Huang X., Han B. 2014. Natural variations and genome-wide association studies in crop plants. Annual Review of Plant Biology 65:531-551. DOI: 10.1146/annurev-arplant-050213035715.

Isobe SN., Hirakawa H., Sato S., Maeda F., Ishikawa M., Mori T., Yamamoto Y., Shirasawa K., Kimura M., Fukami M., Hashizume F., Tsuji T., Sasamoto S., Kato M., Nanri K., Tsuruoka H., Minami C., Takahashi C., Wada T., Ono A., Kawashima K., Nakazaki N., Kishida Y., Kohara M., Nakayama S., Yamada M., Fujishiro T., Watanabe A., Tabata S. 2013. Construction of an integrated high density simple sequence repeat linkage map in cultivated strawberry (Fragaria $\times$ ananassa) and its applicability. DNA research: an international journal for rapid publication of reports on genes and genomes 20:79-92. DOI: 10.1093/dnares/dss035.

Langmead B., Trapnell C., Pop M., Salzberg SL. 2009. Ultrafast and memory-efficient alignment of short DNA sequences to the human genome. Genome Biology 10:R25. DOI: 10.1186/gb-2009-10-3-r25.

Lerceteau-Köhler E., Guérin G., Laigret F., Denoyes-Rothan B. 2003. Characterization of mixed disomic and polysomic inheritance in the octoploid strawberry (Fragaria $\times$ ananassa) using AFLP mapping. Theoretical and Applied Genetics 107:619-628. DOI: 10.1007/s00122-003-1300-6.

Li H., Handsaker B., Wysoker A., Fennell T., Ruan J., Homer N., Marth G., Abecasis G., Durbin R. 2009. The Sequence Alignment/Map format and SAMtools. Bioinformatics 25:20782079. DOI: 10.1093/bioinformatics/btp352. 
433

434

435

436

437

438

439

440

441

442

443

444

445

446

447

448

449

450

451

452

453

454

455

456

457

458

459

460

461

462

463

464

465

466

467

468

469

470

471
Liston A., Cronn R., Ashman T-L. 2014. Fragaria: A genus with deep historical roots and ripe for evolutionary and ecological insights. American Journal of Botany 101:1686-1699. DOI: $10.3732 /$ ajb.1400140.

Mahoney LL., Sargent DJ., Abebe-Akele F., Wood DJ., Ward JA., Bassil NV., Hancock JF., Folta KM., Davis TM. 2016. A high-density linkage map of the ancestral diploid strawberry constructed with single nucleotide polymorphism markers from the IStraw 90 array and genotyping by sequencing. The Plant Genome 9:0. DOI: 10.3835/plantgenome2015.08.0071.

McCallum S., Graham J., Jorgensen L., Rowland LJ., Bassil NV., Hancock JF., Wheeler EJ., Vining K., Poland JA., Olmstead JW., Buck E., Wiedow C., Jackson E., Brown A., Hackett CA. 2016. Construction of a SNP and SSR linkage map in autotetraploid blueberry using genotyping by sequencing. Molecular Breeding 36:1-24. DOI: 10.1007/s11032-016-0443-5.

Molina-Hidalgo FJ., Franco AR., Villatoro C., Medina-Puche L., Mercado JA., Hidalgo MA., Monfort A., Caballero JL., Muñoz-Blanco J., Blanco-Portales R. 2013. The strawberry $($ Fragaria $\times$ ananassa) fruit-specific rhamnogalacturonate lyase 1 (FaRGLyase1) gene encodes an enzyme involved in the degradation of cell-wall middle lamellae. Journal of Experimental Botany 64:1471-1483. DOI: 10.1093/jxb/ers386.

Nagano S., Shirasawa K., Hirakawa H., Maeda F., Ishikawa M., Isobe SN. 2017. Discrimination of candidate subgenome-specific loci by linkage map construction with an S1 population of octoploid strawberry (Fragaria $\times$ ananassa). BMC Genomics 18:374. DOI: 10.1186/s12864-017-3762-y.

Njuguna W., Liston A., Cronn R., Ashman T-L., Bassil N. 2013. Insights into phylogeny, sex function and age of Fragaria based on whole chloroplast genome sequencing. Molecular Phylogenetics and Evolution 66:17-29. DOI: 10.1016/j.ympev.2012.08.026.

Poland JA., Brown PJ., Sorrells ME., Jannink J-L. 2012. Development of high-density genetic maps for barley and wheat using a novel two-enzyme genotyping-by-sequencing approach. PLOS ONE 7:e32253. DOI: 10.1371/journal.pone.0032253.

Qiao Q., Xue L., Wang Q., Sun H., Zhong Y., Huang J., Lei J., Zhang T. 2016. Comparative transcriptomics of strawberries (Fragaria spp.) provides insights into evolutionary patterns. Frontiers in Plant Science 7. DOI: 10.3389/fpls.2016.01839.

Rousseau-Gueutin M., Gaston A., Aïnouche A., Aïnouche ML., Olbricht K., Staudt G., Richard L., Denoyes-Rothan B. 2009. Tracking the evolutionary history of polyploidy in Fragaria L. (strawberry): New insights from phylogenetic analyses of low-copy nuclear genes. Molecular Phylogenetics and Evolution 51:515-530. DOI: 10.1016/j.ympev.2008.12.024.

Rousseau-Gueutin M., Lerceteau-Köhler E., Barrot L., Sargent DJ., Monfort A., Simpson D., Arús P., Guérin G., Denoyes-Rothan B. 2008. Comparative genetic mapping between octoploid and diploid Fragaria species reveals a high level of colinearity between their 
507

508

509

genomes and the essentially disomic behavior of the cultivated octoploid strawberry. Genetics 179:2045-2060. DOI: 10.1534/genetics.107.083840.

Russell J., Hackett C., Hedley P., Liu H., Milne L., Bayer M., Marshall D., Jorgensen L., Gordon S., Brennan R. 2014. The use of genotyping by sequencing in blackcurrant (Ribes nigrum): developing high-resolution linkage maps in species without reference genome sequences. Molecular Breeding 33:835-849. DOI: 10.1007/s11032-013-9996-8.

Saintenac C., Jiang D., Wang S., Akhunov E. 2013. Sequence-based mapping of the polyploid wheat genome. G3\&amp;\#58; Genes|Genomes|Genetics 3:1105-1114. DOI: $10.1534 / \mathrm{g} 3.113 .005819$.

Sánchez-Sevilla JF., Horvath A., Botella MA., Gaston A., Folta K., Kilian A., Denoyes B., Amaya I. 2015. Diversity Arrays Technology (DArT) marker platforms for diversity analysis and linkage mapping in a complex crop, the octoploid cultivated strawberry (Fragaria $\times$ ananassa). PLoS ONE 10. DOI: 10.1371/journal.pone.0144960.

Sargent DJ., Passey T., Šurbanovski N., Girona EL., Kuchta P., Davik J., Harrison R., Passey A., Whitehouse AB., Simpson DW. 2012. A microsatellite linkage map for the cultivated strawberry (Fragaria $\times$ ananassa) suggests extensive regions of homozygosity in the genome that may have resulted from breeding and selection. Theoretical and Applied Genetics 124:1229-1240. DOI: 10.1007/s00122-011-1782-6.

Sargent DJ., Yang Y., Šurbanovski N., Bianco L., Buti M., Velasco R., Giongo L., Davis TM. 2016. HaploSNP affinities and linkage map positions illuminate subgenome composition in the octoploid, cultivated strawberry (Fragaria $\times$ ananassa). Plant Science: An International Journal of Experimental Plant Biology 242:140-150. DOI: 10.1016/j.plantsci.2015.07.004.

Shields M., Davis T. 2017. Implementation of the IStraw90 Axiom SNP Array Genotyping Platform for Genetic Linkage Map Construction in the Diploid Strawberry Model Species, Fragaria vesca. Poster presentation. Plant and Animal Genome Conference XXV, San Diego, California.

Spigler RB., Ashman T-L. 2011. Sex ratio and subdioecy in Fragaria virginiana: the roles of plasticity and gene flow examined. New Phytologist 190:1058-1068. DOI: 10.1111/j.1469-8137.2011.03657.x.

Spindel J., Begum H., Akdemir D., Virk P., Collard B., Redoña E., Atlin G., Jannink J-L., McCouch SR. 2015. Genomic selection and association mapping in rice (Oryza sativa): effect of trait genetic architecture, training population composition, marker number and statistical model on accuracy of rice genomic selection in elite, tropical rice breeding lines. PLoS genetics 11:e1004982. DOI: 10.1371/journal.pgen.1004982.

Spindel J., Wright M., Chen C., Cobb J., Gage J., Harrington S., Lorieux M., Ahmadi N., McCouch S. 2013. Bridging the genotyping gap: using genotyping by sequencing (GBS) to add high-density SNP markers and new value to traditional bi-parental mapping and 
510

511

512

513

514

515

516

517

518

519

520

521

522

523

524

525

526

527

528

529

530

531

532

533

534

535

536

537

538

539

540

541

542

543

544

breeding populations. Theoretical and Applied Genetics 126:2699-2716. DOI:

10.1007/s00122-013-2166-x.

Stamatakis A. 2006. RAxML-VI-HPC: maximum likelihood-based phylogenetic analyses with thousands of taxa and mixed models. Bioinformatics (Oxford, England) 22:2688-2690. DOI: 10.1093/bioinformatics/btl446.

Tennessen JA., Govindarajulu R., Ashman T-L., Liston A. 2014a. Evolutionary origins and dynamics of octoploid strawberry subgenomes revealed by dense targeted capture linkage maps. Genome Biology and Evolution:evu261. DOI: 10.1093/gbe/evu261.

Tennessen JA., Govindarajulu R., Ashman T-L., Liston A. 2014b. Evolutionary origins and dynamics of octoploid strawberry subgenomes revealed by dense targeted capture linkage maps. Genome Biology and Evolution:evu261. DOI: 10.1093/gbe/evu261.

Tennessen JA., Govindarajulu R., Liston A., Ashman T-L. 2016. Homomorphic ZW chromosomes in a wild strawberry show distinctive recombination heterogeneity but a small sex-determining region. New Phytologist 211:1412-1423. DOI: 10.1111/nph.13983.

Van Ooijen. 2006. JoinMap ${ }^{\circledR}$ 4, Software for the calculation of genetic linkage maps in experimental populations. Kyazma B.V., Wageningen, Netherlands.

Ward JA., Bhangoo J., Fernández-Fernández F., Moore P., Swanson J., Viola R., Velasco R., Bassil N., Weber CA., Sargent DJ. 2013. Saturated linkage map construction in Rubus idaeus using genotyping by sequencing and genome-independent imputation. BMC Genomics 14:2. DOI: 10.1186/1471-2164-14-2.

Zorrilla-Fontanesi Y., Cabeza A., Domínguez P., Medina JJ., Valpuesta V., Denoyes-Rothan B., Sánchez-Sevilla JF., Amaya I. 2011. Quantitative trait loci and underlying candidate genes controlling agronomical and fruit quality traits in octoploid strawberry (Fragaria $\times$ ananassa). Theoretical and Applied Genetics 123:755-778. DOI: 10.1007/s00122-011$1624-6$.

\section{Tables}

Table 1. Sequence data obtained from the parents on the Illumina HiSeq2000. Numbers of sequence reads aligning to the Fragaria vesca ssp. bracteata genome assembly, and the SNPs called from those reads by the POLiMAPS pipeline, are indicated

\begin{tabular}{|c|c|c|c|c|c|}
\hline Parent & $\begin{array}{c}\text { No. } \\
\text { Reads } \\
\text { with } \\
\text { Barcode }\end{array}$ & $\begin{array}{c}\text { Total } \\
\text { Basepairs }\end{array}$ & $\begin{array}{c}\text { Reads } \\
\text { aligning } \\
\text { to Fvb }\end{array}$ & $\begin{array}{l}\text { Polymorphic } \\
\text { SNPs per } \\
\text { Parent }\end{array}$ & $\begin{array}{c}\text { Number of SNPs } \\
\text { Mapped }\end{array}$ \\
\hline
\end{tabular}




\begin{tabular}{lrrrrr}
\hline \multicolumn{7}{c}{$\begin{array}{c}\text { and } \\
\text { ApeKI } \\
\text { cut site }\end{array}$} & & & \\
\\
\hline 'Holiday' & $2,113,031$ & $135,233,984$ & $1,304,324$ & 735 & \\
'Korona' & $\underline{2,892,457}$ & $\underline{185,117,248}$ & $\underline{1,709,640}$ & $\underline{1,096}$ & $\underline{305 / 2136}$ \\
\hline 'Tribute' & $1,996,412$ & $127,770,368$ & $1,194,954$ & $\underline{468}$ & $337 / 1163$ \\
'Honeoye' & $\underline{2,892,682}$ & $\underline{185,131,648}$ & $\underline{1,754,838}$ & $\underline{468}$ & \\
\hline 'Redgauntlet' & $2,495,364$ & $159,703,296$ & $1,474,896$ & 1,253 & $722 / 3190$ \\
'Hapil' & $1,536,347$ & $98,326,208$ & 992,053 & 1,215 & \\
\hline
\end{tabular}

545

546

547 548

549

550

551

Table 2. Numbers of JoinMap groups per parent, first round (before phylogenetic analysis) and second round (after group merging).

\begin{tabular}{lllllllllllllll} 
& \multicolumn{3}{c}{ Fvb1 } & \multicolumn{2}{c}{ Fvb2 } & \multicolumn{2}{c}{ Fvb3 } & \multicolumn{2}{c}{ Fvb4 } & \multicolumn{2}{c}{ Fvb5 } & \multicolumn{2}{c}{ Fvb6 } & \multicolumn{2}{c}{ Fvb7 } \\
Parent & $1^{\text {st }}$ & $2^{\text {nd }}$ & $1^{\text {st }}$ & $2^{\text {nd }}$ & $1^{\text {st }}$ & $2^{\text {nd }}$ & $1^{\text {st }}$ & $2^{\text {nd }}$ & $1^{\text {st }}$ & $2^{\text {nd }}$ & $1^{\text {st }}$ & $2^{\text {nd }}$ & $1^{\text {st }}$ & $2^{\text {nd }}$ \\
\hline Tribute & 5 & 4 & 4 & 4 & 6 & 5 & 4 & 3 & 4 & 4 & 4 & 4 & 2 & 3 \\
Honeoye & 4 & 4 & 4 & 5 & 5 & 4 & 5 & 4 & 5 & 4 & 6 & 4 & 4 & 4 \\
Holiday & 4 & 4 & 6 & 4 & 5 & 5 & 4 & 4 & 6 & 4 & 9 & 6 & 4 & 2 \\
Korona & 6 & 4 & 9 & 5 & 9 & 5 & 6 & 4 & 9 & 6 & 6 & 4 & 4 & 3 \\
Redgauntlet & 5 & 4 & 8 & 6 & 5 & 4 & 5 & 4 & 5 & 6 & 5 & 5 & 6 & 5 \\
Hapil & 5 & 4 & 5 & 4 & 5 & 4 & 5 & 4 & 5 & 5 & 7 & 6 & 5 & 4 \\
\hline
\end{tabular}

Table 3. JoinMap groups sub-genome assignment: $F$. vesca-like (v), F. iinumae-like (i), unassigned $(\mathrm{u})$, for each parent.

Fvb1 Fvb2

Fvb3

Fvb4

Fvb5

Fvb6

Fvb7

\begin{tabular}{lccccccc}
\hline Parent & Av, Bi, B12 & $\mathbf{A v}, \mathbf{B i}, \mathbf{B 1 2}$ & $\mathbf{A v}, \mathbf{B i}, \mathbf{B 1 2}$ & $\mathbf{A v}, \mathbf{B i}, \mathbf{B 1 2}$ & $\mathbf{A v}, \mathbf{B i}, \mathbf{B 1 2}$ & $\mathbf{A v}, \mathbf{B i}, \mathbf{B 1 2}$ & $\mathbf{A v}, \mathbf{B i}, \mathbf{B 1 2}$ \\
\cline { 1 - 1 } & $1,0,3$ & $2,0,2$ & $1,0,4$ & $1,1,1$ & $0,0,4$ & $0,0,4$ & $1,0,2$ \\
Tribute & $1,0,3$ & $1,0,4$ & $0,0,4$ & $1,2,1$ & $2,0,2$ & $0,0,4$ & $1,1,2$ \\
Honeoye & $1,0,3$ & $0,1,3$ & $5,0,0$ & $0,1,3$ & $1,0,3$ & $0,0,6$ & $0,0,2$ \\
Holiday & $1,0,3$ & $1,0,4$ & $0,2,3$ & $0,1,3$ & $1,0,5$ & $0,0,4$ & $1,1,1$ \\
Korona & $1,0,3$ & $1,0,5$ & $0,1,3$ & $1,1,2$ & $1,0,5$ & $0,0,5$ & $3,1,1$ \\
Redgauntlet & $1,0,3$ & $1,0,3$ & $0,1,3$ & $0,1,3$ & $1,0,4$ & $1,0,5$ & $1,1,2$ \\
Hapil & & & & & &
\end{tabular}

\section{Figures}

Figure 1. Linkage group relative chromosome positions on the Fragaria vesca reference genome. Linkage groups are color-coded to reflect their relative proximity to ancestral species on phylogenetic trees. Undetermined groups did not clearly cluster with any ancestral species. A. Crossing parent 'Holiday' B. Crossing parent 'Korona'.

A. 


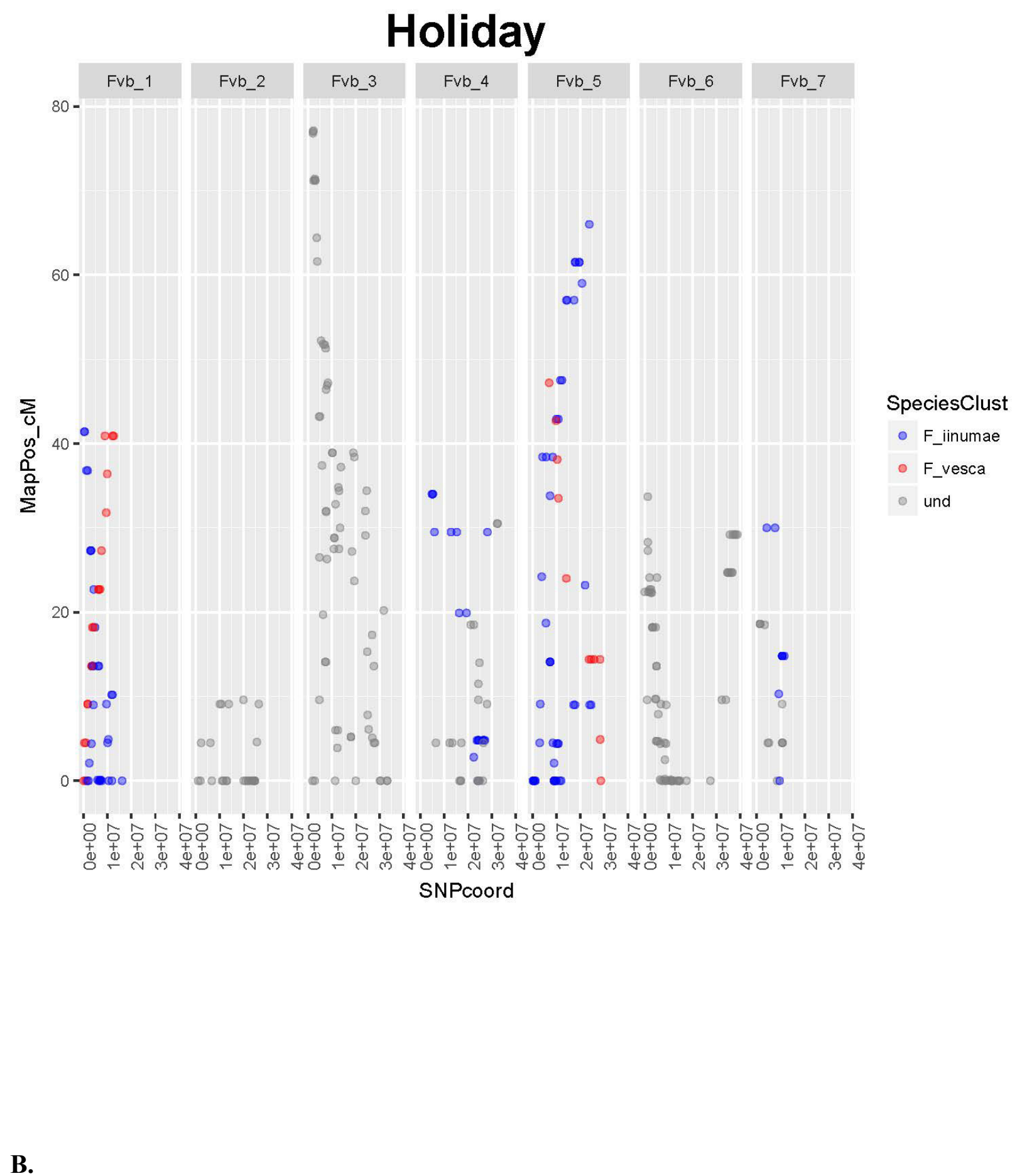

563

564

565

566

567

568

569

570

571

572

B. 


\section{Korona}

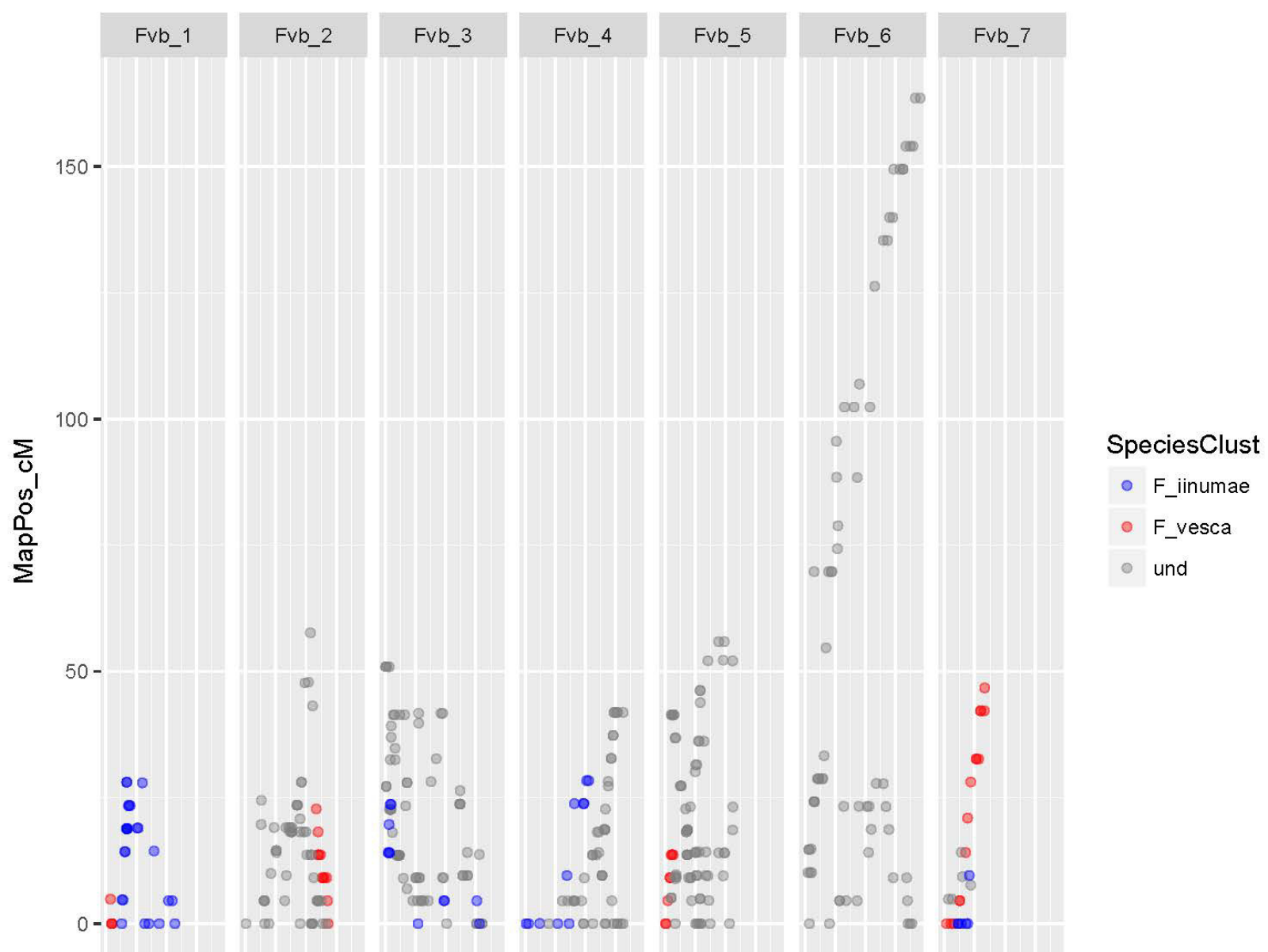

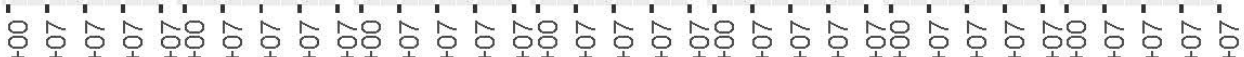

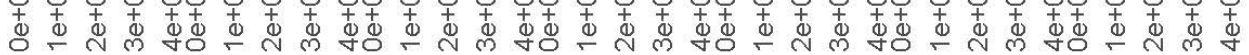


589 Figure 2. Numbers of SNPs from each parent represented on linkage groups aligning to Fragaria 590 vesca chromosomes.

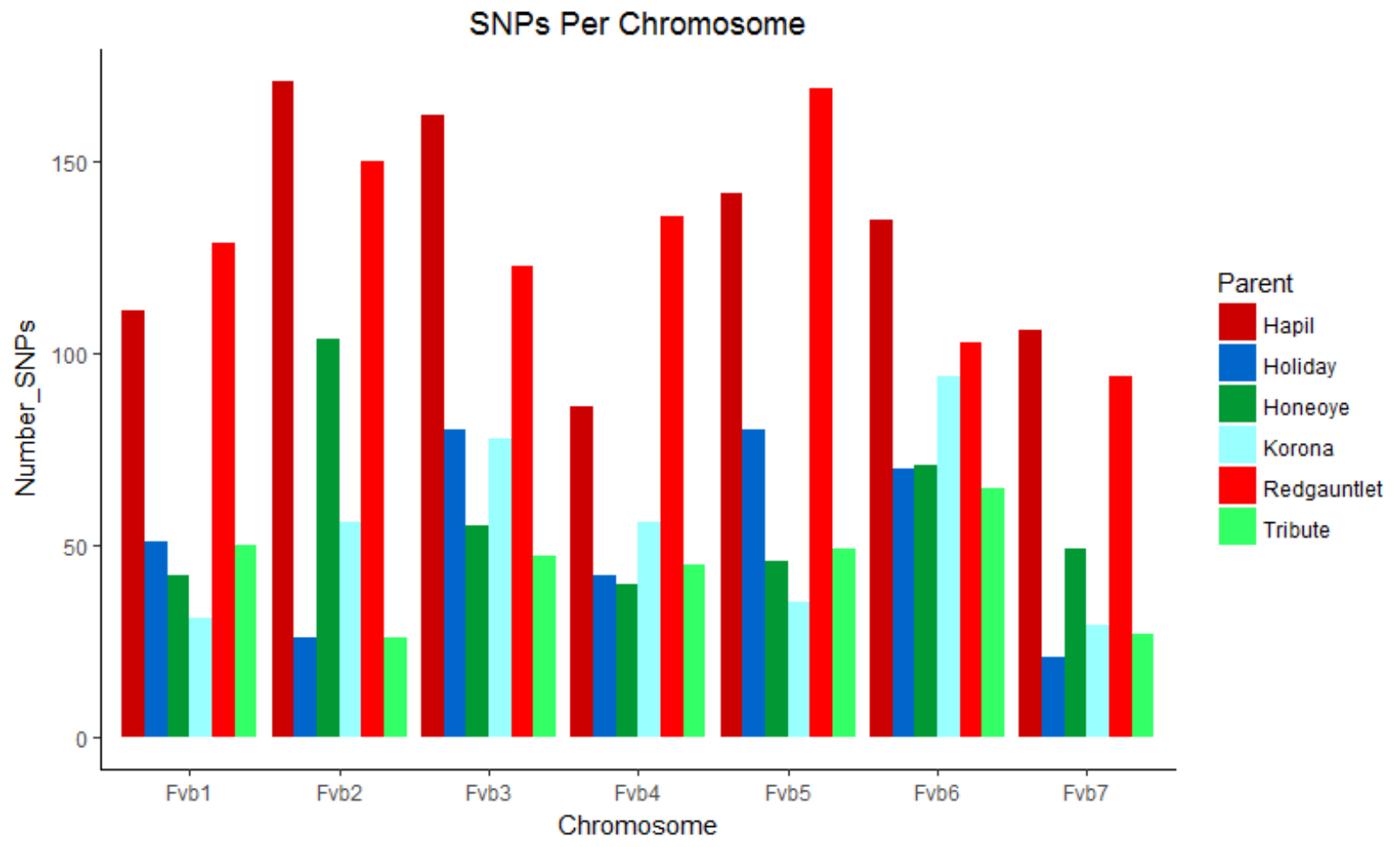

591

593

594

595

596

597

598

599

600

601

602

603

604

605

606

607

608

609

610

611

612

613

614 
615 Figure 3. Cladograms representing linkage groups that aligned to $F$. vesca chromosomes Fvb1 616 (A) and Fvb6 (B). Fvb1 shows a clear $F$. vesca -like clade (A), while Fvb6 lacks a distinct $F$. 617 vesca-like clade (B).

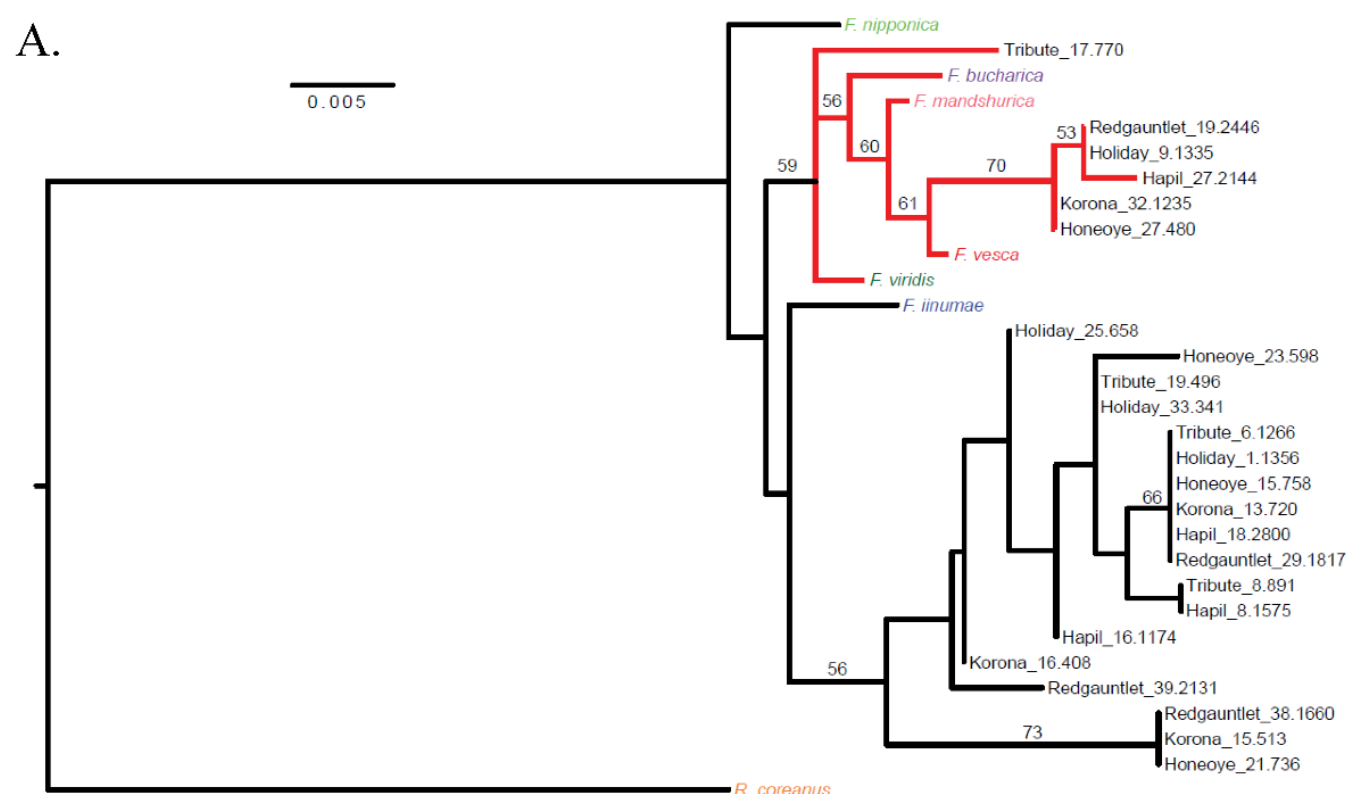

B.

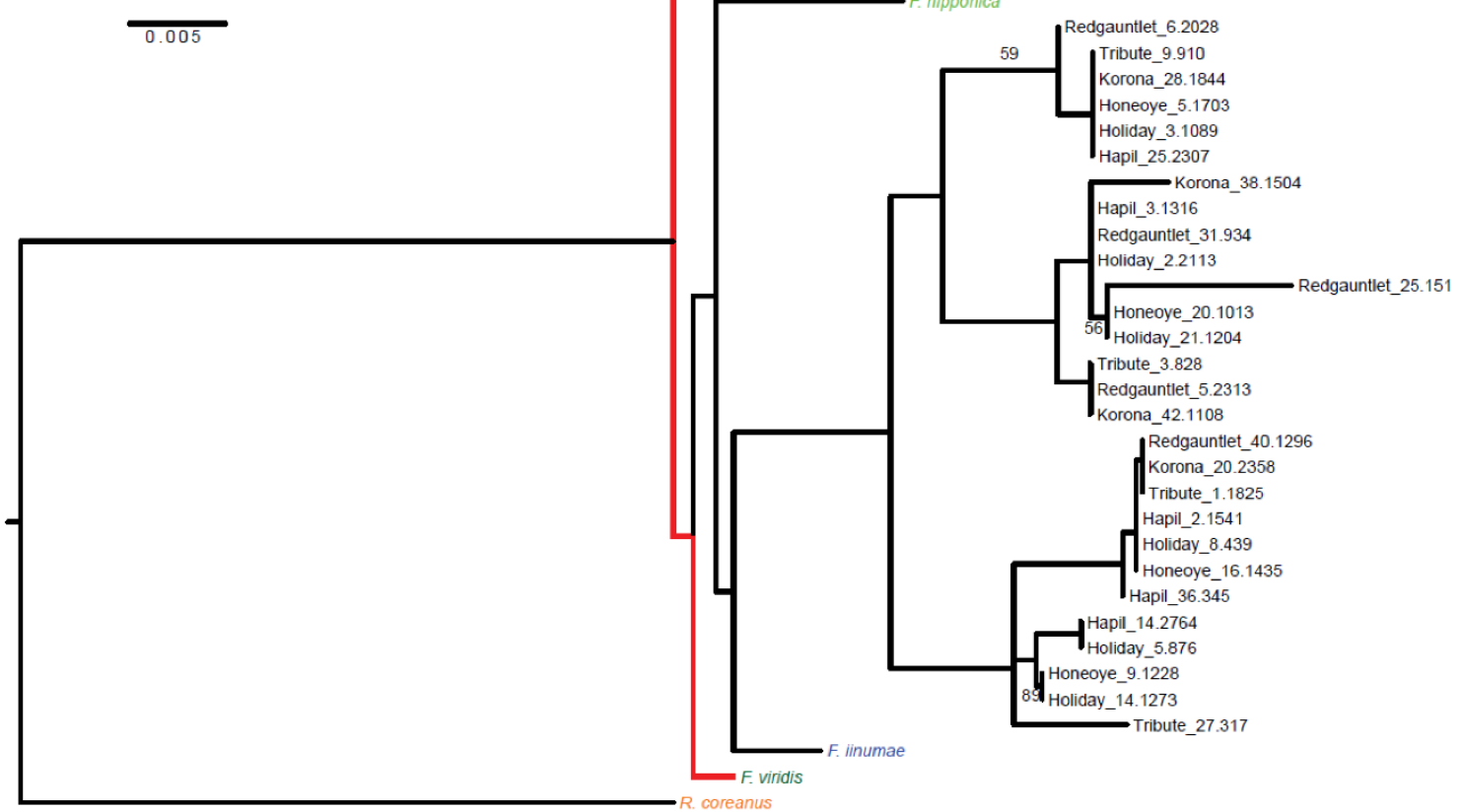


619 Figure 4. Alignment of the integrated map of 'Holiday' LG 6 to the physical map for that 620 chromosome from the Fvb assembly to LG 6 based on SNP data from the 90K Axiom array.

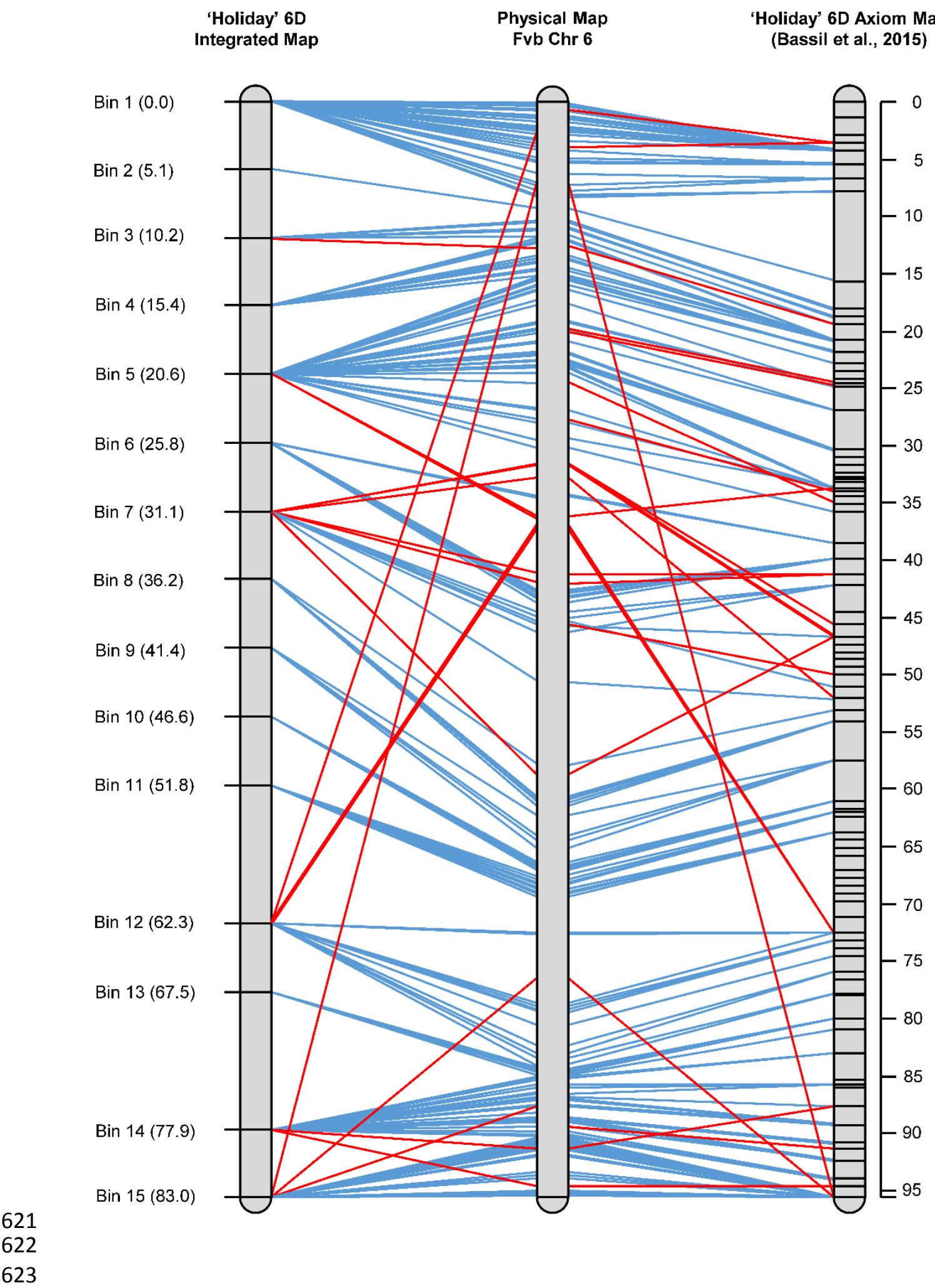


624 Supplemental File 1. JoinMap linkage groups with markers derived from parents 'Holiday' and 625 'Korona' that aligned to $F v b$ chromosomes.

626

627 Supplemental File 2. JoinMap linkage groups with markers derived from parents 'Tribute' and 628 'Honeoye' that aligned to $F v b$ chromosomes.

629

630 Supplemental File 3. JoinMap linkage groups with markers derived from parents 'Redgauntlet' 631 and 'Hapil' that aligned to $F v b$ chromosomes.

632 